\title{
Permanent Section Analysis of Sentinel Lymph Nodes in Breast Cancer
}

\author{
Mahsa Ahadi, ${ }^{1}$ Hanieh Zham, ${ }^{1}$ Mahyar Kiafar, ${ }^{1}$ Sonia Falahkhorsand, ${ }^{1}$ Mohammad Esmaeil Akbari, ${ }^{1}$ \\ Mahasti Amoui, ${ }^{2}$ Maryam Baikpour, ${ }^{1}$ Maryam Khayamzadeh, ${ }^{1}$ and Afshin Moradi ${ }^{1, *}$ \\ ${ }^{1}$ Cancer Research Center, Shohada-e Tajrish Hospital, Shahid Beheshti University of Medical Sciences, Tehran, Iran \\ ${ }^{2}$ Department of Nuclear Medicine, Shohada-e Tajrish Hospital, Shahid Beheshti University of Medical Sciences, Tehran, Iran \\ "Corresponding author: Afshin Moradi, Pathology Department, Shohada-e Tajrish Hospital, Tajrish Square, Tehran, Iran. Tel: +98-2122718049, E-mail: \\ dr.afshin.moradi@gmail.com
}

Received 2017 April 02; Revised 2017 April 25; Accepted 2017 September 05.

\begin{abstract}
Background: Axillary lymph node dissection is still the standard procedure performed for patients with breast cancer with clinically palpable axillary nodes or positive nodes confirmed by various methods. Sentinel lymph node (SLN) biopsy has recently emerged as a substitute for axillary dissection in patients with clinically negative axillary nodes and can spare some of the patients the need for re-operative lymphadenectomy.

Methods: In this descriptive retrospective study, patients with definite diagnosis of breast cancer treated in the Shohada-e Tajrish hospital during 2008 - 2014 were identified. Of these patients, subjects who had undergone intraoperative frozen section analysis of the SLN biopsy and had a report of a permanent section analysis were included. Age and the results of frozen section and permanent section analyses were extracted from the medical records and proceeded by SPSS statistical software v.22.

Results: A total of 135 eligible women were included with the mean age of $48.85 \pm 11.62$ years. Frozen section analysis of SLN biopsy accurately identified 36 positive cases and 89 negative cases with an overall accuracy of $92.6 \%$. Sensitivity, specificity, positive predictive value (PPV) and negative predictive value (NPV) were calculated to be $81.8 \%, 97.8 \%, 94.7 \%$ and $91.7 \%$, respectively. The correlation between 2 analyses was found to be statistically significant (Pearson's $\mathrm{R}=0.830, \mathrm{P}<0.001$ ).

Conclusions: Intraoperative frozen section analysis of SLN biopsy in patients with breast cancer is a useful method, which can determine the need for immediate axillary dissection and can spare the patients with positive results, the need for reoperation.
\end{abstract}

Keywords: Frozen Section, Permanent Section, Sentinel Lymph Node, Breast Cancer

\section{Background}

Breast cancer is the most frequently diagnosed cancer all around the world as well as the leading cause of death in women (1).

The most important independent factor that can predict the survival and risk of recurrence in patients with breast cancer is the histopathologic status of axillary lymph nodes (2). Accordingly, axillary lymph node dissection has been considered a routine part of the management for patients with early breast cancer. This method affects disease control, has a high prognostic value, and guides treatment selection for the patients (3). However, it causes anatomic disruption and can be associated with complications, such as lymphedema, nerve injury, and shoulder dysfunction, which can diminish functionality of the patients and affect the quality of their life (4).
Axillary lymph node dissection is still the standard procedure performed for women with clinically palpable axillary nodes or positive nodes confirmed by methods, such as ultrasound guided fine needle aspiration (5). Recently emerged as an accepted substitute for axillary dissection in staging breast cancer in patients with clinically negative axillary nodes, sentinel lymph node(SLN) biopsy yields a high accuracy with a low false negative rate and is associated with less morbidity while performed by an experienced physician $(6,7)$.

The pathologic evidence of axillary metastasis will only be present in approximately 30\% of patients with T1-2, no breast carcinomas $(6,8)$. Although frozen section is known to be less accurate than permanent section analysis, it can help avoid a second surgical intervention for axillary lymph node dissection in patients with positive metastases (9). 
SLN has been shown, by various studies, to have a high detection rate and an acceptable accuracy for predicting axillary lymph node status (10-13); however, few studies have compared its compatibility with the results of permanent section analysis in breast cancer. Therefore, the present study aims at investigating the compatibility of intraoperative frozen section analysis with permanent section analysis of SLN in patients who had been referred to Shohadaye Tajrish Hospital during 2008 - 2014 with a diagnosis of breast cancer.

\section{Methods}

In this descriptive retrospective study, patients with definite diagnosis of breast cancer, made through histopathological assessment, were identified by reviewing the archives of pathology reports documented in the department of pathology of Shohadaye Tajrish hospital during 2008 - 2014. Of these patients, those who had undergone intraoperative frozen section analysis of the SLN biopsy and had had a report of a permanent section analysis were included as the study population, using a convenience sampling method.

Hence, the inclusion criteria of the study included making a definite diagnosis of breast cancer made through histopathological assessment, having undergone intraoperative frozen section analysis of the SLN biopsy, and having the patient's permanent section analysis report. Incomplete medical record was also considered the exclusion criteria.

For pathological assessments, lymph nodes were obtained fresh, and after sampling, were frozen as quickly as possible at temperature $-20^{\circ} \mathrm{C}$ to $-30^{\circ} \mathrm{C}$. Then, the prepared slides were stained according to the standard protocol of Hematoxylin and Eosin ( $\mathrm{H}$ and $\mathrm{E}$ ) staining.

The sentinel nodes would be considered negative on frozen section if we were not able to find tumoral involvement in at least three-step sections stained with H\&E. Immune staining for Keratin was not performed on the specimens during intraoperative consultation.

The data of frozen section and permanent section analyses were extracted from the medical records and processed by SPSS (statistical software v.22) (14). The results of analyses were recorded as dichotomous variables of positive and negative states and their compatibility were compared, using Pearson's correlation analysis. Sensitivity, specificity, negative predictive value and positive predictive value were, then, calculated accordingly. A P value of less than 0.05 was considered statistically significant in all analyses.

The patients' information was remained confidential throughout the survey and the extracted data were used anonymously. The study protocol was evaluated and approved by the ethics committee of Shahid Beheshti University of Medical Sciences.

\section{Results}

A total of 135 eligible women diagnosed with breast cancer and recorded frozen section analysis of SLN and permanent section analysis were included in this study. The mean age of the subjects was calculated to be $48.85 \pm 11.62$ years with a minimum of 23 and a maximum of 79 years. Figure 1 depicts the age distribution of the study population.

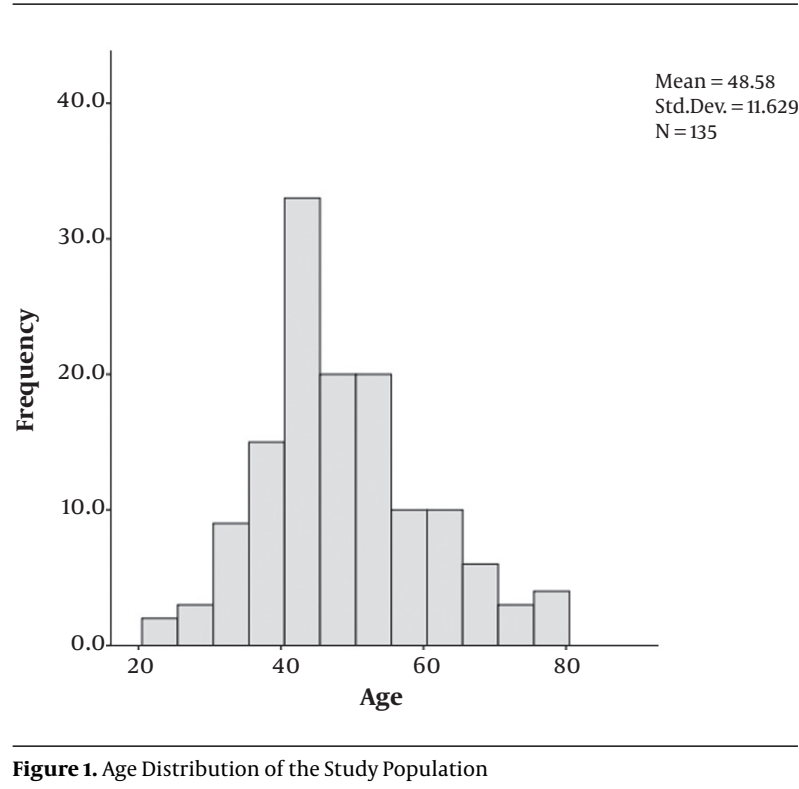

The frozen section analysis of SLN biopsies was positive in 38 patients (28.1\%) and negative in 97 (71.9\%), while the results of permanent section analysis was positive in 44 (32.6\%) and negative in 91 subjects (67.4\%) (Table 1).

Table 1. Results of the Study

\begin{tabular}{|cc}
\hline Variable & Number $/$ Mean \\
\hline Age (mean \pm standard deviation) & $48.85 \pm 11.63$ years \\
\hline Frozen section analysis & \\
\hline Positive & $38(28.1 \%)$ \\
\hline Negative & $97(71.9 \%)$ \\
\hline Permanent section analysis & \\
\hline Positive & $44(32.6 \%)$ \\
\hline Negative & $91(67.4 \%)$ \\
\hline
\end{tabular}


Table 2 presents the correlation between 2 histological analyses performed for the patients. Based on these results, frozen section analysis of SLN biopsy accurately identified 36 positive cases and 89 negative cases; thus, the overall accuracy of this method is $92.6 \%$. Sensitivity, specificity, positive predictive value (PPV) and negative predictive value (NPV) were calculated to be $81.8 \%, 97.8 \%, 94.7 \%$, and $91.7 \%$, respectively. The false negative rate (1-sensitivity) and false negative predictive value (1-NPV) were also found to be $18.2 \%$ and $8.3 \%$, respectively.

The correlation between these 2 analyses was evaluated and it was found to be statistically significant with a Pearson's correlation coefficient of $0.830(\mathrm{P}<0.001)$.

Table 2. Correlation Between Frozen and Permanent Sections

\begin{tabular}{l|c|c|c|c}
\hline \multirow{2}{*}{ Frozen section } & & \multicolumn{2}{|c|}{ Permanent Section } & \multirow{2}{*}{ Total } \\
\cline { 3 - 5 } & & Positive & Negative & \\
\hline \multirow{2}{*}{ Total } & Positive & 36 & 2 & 38 \\
\cline { 2 - 5 } & Negative & 8 & 89 & 97 \\
\hline
\end{tabular}

\section{Discussion}

Based on the results of the present study, a significant correlation was found between frozen section and permanent section analyses. Although the diagnostic characteristics calculated for frozen section analysis confirm the usefulness of this method in patients with breast cancer, the small risk of false positive results $(1.5 \%)$ should also be recognized. However, 81.8\% (36 out of 44 ) of the patients who are spared the need for reoperation seem to outweigh the mentioned small risk.

In recent decades, a growing tendency towards less invasive procedures has been observed in the treatment of breast cancer inasmuch as currently more than 50\% of these patients undergo breast-conserving surgeries instead of extensive total and radical mastectomies (15). Accordingly, in the early stages of cancer axillary lymph node, biopsy and pathologic evaluation of the specimens are performed as a superior method to completion dissection of these nodes, which is accompanied by multiple debilitating complications. In this setting, patients with positive lymph nodes require re-operative lymphadenectomy. To spare the need for such interventions in these patients, intraoperative frozen section analysis of SLN biopsy is introduced as an alternative method so that patients with positive SLN biopsies would undergo lymphadenectomy in their first surgery and would not need a reoperation (6, 7).
Table 3 presents the results of previous studies on the accuracy of SLN frozen section analysis in breast cancer. As can be seen, discrepancies are observed among the results of these studies. These differences can be attributed to the various clinical and pathological factors that affect the accuracy of frozen section analysis. For instance, nodal metastases from infiltrating ductal carcinomas are generally considered easier to visualize than those from invasive lobular carcinomas $(9,16)$. The tumor size is another factor that might affect the accuracy; the false negative rate might decrease with increasing tumor size (17). Technical issues can also affect the results yielded from frozen section analysis. Although the differences can be minimized by employing the proper techniques and appropriate experience, some tissues still may be lost during the frozen section processes and cause the diagnosis to be missed due to a suboptimal technique $(16,18-21)$. The number of frozen section levels examined is another factor that can influence the accuracy of the procedure (16). An increase in sampling nodal sections can also improve the accuracy and sensitivity of frozen section analysis, but it may be impractical, since it consumes much more time than the routine protocol for this means $(22,23)$. And finally, expertise of the operator is also of utmost importance in the results of analyses $(16,18)$.

Despite the discrepancies observed among these studies, all of them are indicative of the fact that frozen section analysis of SLN is generally accurate for breast cancer and can be used to determine the need for immediate axillary dissection and it can spare patients with positive results, the need for reoperation.

\section{Acknowledgments}

None declared.

\section{Footnotes}

Authors' Contribution: None declared.

Financial Disclosure: None declared.

Conflict of Interest: None declared.

Funding/Support: None declared. 
Table 3. Review of the Literature on Frozen Section Analysis of Sentinel Lymph Nodes in Breast Cancer

\begin{tabular}{|c|c|c|c|c|}
\hline Authors & Number of Patients & Accuracy & Sensitivity & NPV \\
\hline Cavanese et al. (24) & 96 & $91 \%$ & $73 \%$ & $88 \%$ \\
\hline Turner et al. (16) & 278 & $93.2 \%$ & $73.9 \%$ & $91.6 \%$ \\
\hline Veronesi et al. (22) & 119 & $97 \%$ & $94.5 \%$ & $95.5 \%$ \\
\hline Hingston et al. (25) & 97 & $95 \%$ & $89 \%$ & $96 \%$ \\
\hline Van Diest et al. (19) & 54 & $95 \%$ & $87 \%$ & $93.5 \%$ \\
\hline Zurrida et al. (23) & 192 & $86 \%$ & $68 \%$ & $81 \%$ \\
\hline Weiser et al. (17) & 890 & $89 \%$ & $58 \%$ & $87.3 \%$ \\
\hline Rahusen et al. (18) & 100 & $84 \%$ & $57 \%$ & $80 \%$ \\
\hline Chao et al. (9) & 200 & $92.6 \%$ & $67.9 \%$ & $89.8 \%$ \\
\hline Current study & 135 & $92.6 \%$ & $81.8 \%$ & $91.7 \%$ \\
\hline
\end{tabular}

\section{References}

1. Siegel RL, Miller KD, Jemal A. Cancer statistics, 2016. CA Cancer J Clin. 2016;66(1):7-30. doi: 10.3322/caac.21332. [PubMed: 26742998].

2. Fisher B, Bauer M, Wickerham DL, Redmond CK, Fisher ER, Cruz AB, et al. Relation of number of positive axillary nodes to the prognosis of patients with primary breast cancer. An NSABP update. Cancer. 1983;52(9):1551-7. [PubMed: 6352003].

3. Lin PP, Allison DC, Wainstock J, Miller KD, Dooley WC, Friedman $\mathrm{N}$, et al. Impact of axillary lymph node dissection on the therapy of breast cancer patients. J Clin Oncol. 1993;11(8):1536-44. doi: 10.1200/JCO.1993.11.8.1536. [PubMed: 8336192].

4. Warmuth MA, Bowen G, Prosnitz LR, Chu L, Broadwater G, Peterson $B$, et al. Complications of axillary lymph node dissection for carcinoma of the breast: a report based on a patient survey. Cancer. 1998;83(7):1362-8. [PubMed: 9762937].

5. Haygood CW, Fauci J, Shah M, Huh W. National comprehensive cancer network (NCCN) guidelines for gynecologic malignancies: Where's the beef? Gynecol Oncol. 2014;133(Supp1):143.

6. McMasters KM, Tuttle TM, Carlson DJ, Brown CM, Noyes RD, Glaser RL, et al. Sentinel lymph node biopsy for breast cancer: a suitable alternative to routine axillary dissection in multi-institutional practice when optimal technique is used.J Clin Oncol. 2000;18(13):2560-6. doi: 10.1200/JCO.2000.18.13.2560. [PubMed: 10893287].

7. Cody HS. Sentinel lymph node mapping in breast cancer. Breast Cancer. 1999;6(1):13-22.

8. Gann PH, Colilla SA, Gapstur SM, Winchester DJ, Winchester DP. Factors associated with axillary lymph node metastasis from breast carcinoma: descriptive and predictive analyses. Cancer. 1999;86(8):15119. [PubMed: 10526280].

9. Chao C, Wong SL, Ackermann D, Simpson D, Carter MB, Brown CM, et al. Utility of intraoperative frozen section analysis of sentinel lymph nodes in breast cancer. Am JSurg. 2001;182(6):609-15. [PubMed: 11839325].

10. Celebioglu F, Sylvan M, Perbeck L, Bergkvist L, Frisell J. Intraoperative sentinel lymph node examination by frozen section, immunohistochemistry and imprint cytology during breast surgery-a prospective study. Eur J Cancer. 2006;42(5):617-20. doi: 10.1016/j.ejca.2005.12.003. [PubMed: 16446084].

11. Cserni G, Amendoeira I, Apostolikas N, Bellocq JP, Bianchi S, Boecker $W$, et al. Discrepancies in current practice of pathological evaluation of sentinel lymph nodes in breast cancer. Results of a questionnaire based survey by the European Working Group for
Breast Screening Pathology. J Clin Pathol. 2004;57(7):695-701. doi: 10.1136/jcp.2003.013599. [PubMed: 15220360].

12. Tsujimoto M, Nakabayashi K, Yoshidome K, Kaneko T, Iwase T, Akiyama $\mathrm{F}$, et al. One-step nucleic acid amplification for intraoperative detection of lymph node metastasis in breast cancer patients. Clin Cancer Res. 2007;13(16):4807-16. doi: 10.1158/1078-0432.CCR-06-2512. [PubMed: 17699859].

13. Visser M, Jiwa M, Horstman A, Brink AA, Pol RP, van Diest P, et al. Intraoperative rapid diagnostic method based on CK19 mRNA expression for the detection of lymph node metastases in breast cancer. Int J Cancer. 2008;122(11):2562-7. doi:10.1002/ijc.23451. [PubMed:18324628].

14. Algorithms Chicago: IBM SPSS Inc. . SPSS I. IBM SPSS statistics 22. Chicago: Algorithms Chicago: IBM SPSS Inc.; 2013.

15. Veronesi U, Cascinelli N, Mariani L, Greco M, Saccozzi R, Luini A, et al. Twenty-year follow-up of a randomized study comparing breastconserving surgery with radical mastectomy for early breast cancer. N Engl J Med. 2002;347(16):1227-32. doi: 10.1056/NEJMoa020989. [PubMed: 12393819].

16. Turner RR, Hansen NM, Stern SL, Giuliano AE. Intraoperative examination of the sentinel lymph node for breast carcinoma staging. Am J Clin Pathol. 1999;112(5):627-34. [PubMed:10549249].

17. Weiser MR, Montgomery LL, Susnik B, Tan LK, Borgen PI, Cody HS. Is routine intraoperative frozen-section examination of sentinel lymph nodes in breast cancer worthwhile? Ann Surg Oncol. 2000;7(9):651-5. [PubMed: 11034241].

18. Rahusen FD, Pijpers R, Van Diest PJ, Bleichrodt RP, Torrenga H, Meijer $S$. The implementation of the sentinel node biopsy as a routine procedure for patients with breast cancer. Surgery. 2000;128(1):6-12. doi: 10.1067/msy.2000.107229. [PubMed: 10876178].

19. Van Diest PJ, Torrenga H, Borgstein PJ, Pijpers R, Bleichrodt RP, Rahusen FD, et al. Reliability of intraoperative frozen section and imprint cytological investigation of sentinel lymph nodes in breast cancer. Histopathology. 1999;35(1):14-8. [PubMed:10383709].

20. Viale G, Bosari S, Mazzarol G, Galimberti V, Luini A, Veronesi P, et al. Intraoperative examination of axillary sentinel lymph nodes in breast carcinoma patients. Cancer. 1999;85(11):2433-8. [PubMed: 10357414].

21. Ku NN. Pathologic examination of sentinel lymph nodes in breast cancer. Surg Oncol Clin N Am. 1999;8(3):469-79. [PubMed:10448690].

22. Veronesi U, Paganelli G, Viale G, Galimberti V, Luini A, Zurrida S, et al. Sentinel lymph node biopsy and axillary dissection in breast cancer: results in a large series. J Natl Cancer Inst. 1999;91(4):368-73. [PubMed: 10050871].

23. Zurrida S, Galimberti V, Orvieto E, Robertson C, Ballardini B, Cremonesi $\mathrm{M}$, et al. Radioguided sentinel node biopsy to avoid axillary 
dissection in breast cancer. Ann Surg Oncol. 2000;7(1):28-31. [PubMed: 10674445].

24. Canavese G, Gipponi M, Catturich A, Di Somma C, Vecchio C, Rosato $\mathrm{F}$, et al. Sentinel lymph node mapping opens a new perspective in the surgical management of early-stage breast cancer: a combined approach with vital blue dye lymphatic mapping and radioguided surgery. Semin Surg Oncol. 1998;15(4):272-7. [PubMed: 9829386].

25. Hingston G, Cooke T, Going J, Simon R, Flett M. Accuracy of intraoperative frozen-section analysis of axillary nodes. Br J Surg. 1999;86(8):1092. 